\title{
A comparison of the efficacy, safety, and longevity of two different hyaluronic acid dermal fillers in the treatment of severe nasolabial folds:
}

\section{a multicenter, prospective, randomized, controlled, single-blind, within-subject study}

This article was published in the following Dove Press journal:

Clinical, Cosmetic and Investigational Dermatology

19 December 201।

Number of times this article has been viewed

\section{Greg J Goodman' \\ Phillip Bekhor ${ }^{2}$ \\ Michael Rich ${ }^{3}$ \\ Robert H Rosen ${ }^{4}$ \\ Michael B Halstead ${ }^{5}$ \\ John D Rogers ${ }^{5}$ \\ 'Dermatology Institute of Victoria, South Yarra, Victoria, Australia; ${ }^{2}$ Laser Dermatology, Box Hill, Victoria, Australia; ${ }^{3}$ enRich Dermatology and Cosmetic Surgery Centre, Armadale, Victoria, Australia; ${ }^{4}$ Southderm Pty Ltd, Kogarah, New South Wales, Australia; ${ }^{5}$ Allergan Australia, Gordon, New South Wales, Australia}

Correspondence: Greg J Goodman 8-10 Howitt St, South Yarra 3I4I, Victoria, Australia

Tel +6I 398264966

Fax +6I 398277975

Email gg@div.net.au
Background: Commercially available hyaluronic acid (HA)-based fillers have distinct physicochemical properties related to their specific manufacturing technology, including HA concentration, cross-linking percentage, and particle size. These factors may determine treatment effectiveness, safety, and longevity; however, this requires confirmation in the clinic.

Methods: To compare the efficacy, safety, and longevity of two distinct HA-based dermal fillers in the correction of severe nasolabial folds (NLFs), a $24 \mathrm{mg} / \mathrm{mL}$ smooth gel (Juvederm ULTRA PLUS ${ }^{\text {TM }}$ [JUP]) and a $20 \mathrm{mg} / \mathrm{mL}$ particulate gel (Perlane ${ }^{\circledR}$ [PER]) were injected in a total of 80 normal, healthy subjects using a split face design and were followed for 12 months in this prospective, randomized, controlled, multicenter study.

Results: Both fillers achieved a clinically relevant NLF correction (one point or more improvement, based on a validated NLF severity scale). However, JUP displayed greater longevity, with this correction maintained in a significantly larger percentage of NLFs after 6 months (physician's evaluation) or 9 months (subject's evaluation) and thereafter for the remainder of the study (70\% vs $45 \% ; P=0.0002$ and $62.5 \%$ vs $46.3 \% ; P=0.01$ at month 12 , based on physician and subject assessments, respectively). At month $12,71.4 \%$ of the subjects nominated a preference for the NLF injected with JUP $(P<0.0001)$. Both treatments were well tolerated.

Conclusion: These results suggest that different physicochemical properties of HA-based fillers, associated with distinct manufacturing technologies, may influence treatment longevity in the correction of volume deficits. This may relate to a differential resistance to hyaluronidase and/or free radical degradation as previously documented in vitro.

Keywords: hyaluronic acid, dermal fillers, volume deficit, nasolabial folds, randomized, controlled

\section{Introduction}

Facial appearance is defined by properties of the skin, muscle, the bony anatomy, and the amount and distribution of subcutaneous adipose tissue. Structural changes in these tissues generally become noticeable by the age of 30 in the majority of the population. ${ }^{1,2}$ Loss of bone and soft tissue volume, redistribution of fat, and decreased dermal elasticity and thickness contribute to the formation of wrinkles and folds, which commonly characterize the aging process. ${ }^{3}$ In the case of the mid-face, an age- 
related decline in tensile strength of tissues supporting the nasolabial segment results in flattening and furrowing of the central area of the mid-cheek while displacement medially exaggerates the volume and depth of the nasolabial fold (NLF). ${ }^{4-6}$ Correction of facial lines and wrinkles, as well as restoration and alteration in facial shape, are key approaches to the rejuvenation and enhancement of facial appearance., ${ }^{4,7}$ In addition to surgical treatments, injectable collagen, fat, and hyaluronic acid (HA)-based dermal fillers have been used over the past 20 years to achieve these goals.

$\mathrm{HA}$ is a naturally occurring glycosaminoglycan and a key component of the extracellular matrix in all adult animal tissue. Approximately $50 \%$ of the total HA concentration in the body is located in the dermis. ${ }^{8}$ Due to its high affinity for water, HA plays an integral role in maintenance and regulation of moisture within tissues. ${ }^{9,10}$

Dermal fillers containing native HA polymer chains alone would have little therapeutic utility due to a rapid degradation through the activity of hyaluronidases and free radicals, which are present in high concentrations in the dermis. As a consequence, manufacturing processes associated with production of HA-based dermal fillers are designed to change the physical and chemical properties of the HA, with the objective of improving resistance to this degradation in vivo, in addition to facilitating their safe and efficient administration via a syringe, at an acceptable extrusion force. This outcome is achieved for each of the commercially available HA-based dermal fillers through the application of different proprietary-based manufacturing technologies, leading to distinct properties of these fillers, including variances in HA concentration, the production of particulate or smooth gel masses, and associated differences in the degree of covalent binding of the HA polymers to cross-linking agents such as 1,4-butanediol diglycidal ether (BDDE). ${ }^{10,11}$ Two such fillers are Juvederm ULTRA PLUS ${ }^{\text {TM }}$ (JUP; Allergan Inc, Irvine, CA) and Perlane ${ }^{\circledR}$ (PER; Q-Med AB, Uppsala, Sweden). The final JUP product is a $24 \mathrm{mg} / \mathrm{mL}$ smooth, cohesive gel resulting from a proprietary cross-linking process involving BBDE, which forms a dense HA network. By contrast, the manufacture of PER is characterized by a lower relative degree of cross-linking by BDDE and, notably, a $20 \mathrm{mg} / \mathrm{mL}$ homogenous particulate gel mass resulting from the gel being pushed through a screen during a final production stage to create particles between $750 \mu \mathrm{m}$ and $1000 \mu \mathrm{m} .^{10-13}$

To investigate whether the aforementioned product characteristics imparted a differential resistance to hyaluronidase activity, Sall and Ferard ${ }^{14}$ conducted in vitro evaluation of eleven commercially available gels, including JUP and PER.
In this study, the PER gel was hydrolyzed at a significantly greater rate than for the JUP gel, reflecting a better stability of the latter to this enzymatic degradation. The authors proposed that this greater relative resistance of JUP was due to its higher degree of HA polymer cross-linking, creating a more effective enzymatic barrier. Conversely, a larger HA surface area was available for hydrolysis in the particulate PER gel.

To test whether the differential degradation of these two dermal fillers in vitro would translate into a variance in their clinical performance, subjects with severe NLFs were enrolled into this multicenter, prospective, randomized, single-blind, within-subject ("split-face") study. The primary objective of this study was to compare the efficacy, safety, and, specifically, the longevity of a single administration of JUP versus PER in the correction of NLFs over a period of 12 months.

\section{Methods \\ Subjects}

Healthy male or female subjects aged 30 years or older with fully visible and symmetrical NLFs of a severe presentation, defined as a score of 3 on the five-point validated Physician's Wrinkle Assessment/Nasolabial Fold Photonumeric Rating Scale ("NLF severity scale"), ${ }^{4}$ were eligible for the study. Exclusion criteria included any pre-existing condition that might affect efficacy and/or evaluation of response, including visible scars, active inflammation, infection, cancerous or precancerous lesions, and prior surgical cosmetic procedures in the treatment area. Subjects were excluded if any of the following criteria were met: a history of dermal filler or fat injections within 9 months of randomization, botulinum toxin treatment or mesotherapy within 6 months of randomization, and a history of permanent or semipermanent facial implants in the lower two-thirds of the face. A history of HA hypersensitivity, connective tissue disease, bleeding disorders, use of aspirin during the week preceding the study treatment, concomitant anticoagulant, or antiplatelet therapy were also exclusion criteria. During the study period, the use of antiwrinkle therapies in the area of the NLF or around the mouth was not permitted, with the exception of topical skincare products. Females of childbearing potential were required to have a negative urine pregnancy test and to use adequate contraception while participating in the study.

The study was approved by a central institutional review board and was conducted at four private dermatology clinics in accordance with the applicable Good Clinical Practice 
regulations and guidelines, including the International Conference on Harmonization (ICH) guidelines. All subjects were required to provide written informed consent prior to any study-related procedure. This study is listed on the Australian New Zealand Clinical Trial Registry (ACTRN12609000969268).

\section{Treatment}

In this within-subject comparator study, eligible subjects were randomly assigned to receive a single treatment with either JUP or PER into the right NLF, with the alternative treatment administered into the left NLF. No "touch-ups" were permitted during the study. As a commercial product was used in the trial, the physician investigators were not blinded to the product they were injecting. However, after the initial treatment session, the randomization code was kept in a location separate from the other study documents and was not freely available for review by the investigators. The subjects wore a blindfold during the injection procedure and were thus unaware of which product was injected into each NLF. This information was withheld from them for the entire duration of the 12-month study.

Before administration of the fillers, the treatment areas were prepared with antiseptic solution. The study products were injected into the mid to deep dermis (JUP) or deep dermis (PER), using a 27-gauge needle. The investigators were instructed to use as much product as deemed necessary (up to two syringes of each allocated product) to achieve optimal correction of the NLF. As neither study product contained lidocaine in their formulation, the use of ice before and after the product administration was mandatory (topical or injectable anesthetic agents were not permitted, in order to minimize the potential for the distortion of the NLF region anatomy).

\section{Outcome measures}

The severity of each NLF was determined by the physician at baseline and at the scheduled clinic visits during the course of the study (days 3 and 7 and months 1, 3, 6, 9, and 12), based on the NLF severity scale. This scale ranges from none (" 0 "; "no wrinkle") to extreme ("4"; "very deep wrinkle, redundant fold, overlapped skin"). A validated photonumeric guide was employed for this physician scale, comprising reference photographs that were labeled with descriptors for each of the five NLF severity grades. ${ }^{4}$ All physician investigators were trained to use this scale prior to study initiation.

At the same clinic visits, the study subjects were also asked to assess the severity of each NLF, using the NLF severity scale however without the photonumeric guide.
This self-assessment was always completed prior to and independent of the physician assessment, with the scores documented on separate case report forms.

At the end of the study (month 12), while still blinded to the treatment allocation, subjects were asked to indicate their preferred treatment site (right or left NLF), if any, based on their perception of treatment outcome.

To evaluate the safety and tolerability of the two study treatments, on days 1 through 3 post-treatment, each subject kept a diary to record the degree of bruising, swelling, redness, or pain at the injection site for each NLF. Subjects were also asked to evaluate the degree of pain they experienced at each NLF immediately following treatment (day 1), based on an eleven-point Likert scale, ranging from "no pain" to "worst pain imaginable". The physician investigators also assessed each NLF on the initial two post-treatment follow-up clinic visits (days 3 and 7) for evidence of bruising, swelling, or redness. At these clinic visits, the subjects were asked to record the level of discomfort and tenderness on palpation using a 100-mm visual analog scale (VAS), ranging from "no discomfort (or tenderness)" to "worst discomfort (or tenderness) imaginable".

\section{Statistical analysis}

All subjects receiving the study treatment were included in the efficacy and safety analyses. Missing efficacy data were replaced with those from the last available observation. In this within-subject study design, each patient was his/her own control, with the analysis based on the degree of correction at the left versus the right NLF for each subject, as determined by the independent rating of the physician and the subject over the 12-month post-treatment assessment period, using the validated NLF severity scale. Paired $t$-tests were used to evaluate the significance of continuous outcomes, and McNemar's test was used to assess categorical outcomes. All statistical tests were two-sided, and $P$ values $<0.05$ were considered statistically significant.

A clinically meaningful treatment effect and the primary outcome variable for this study were defined as an improvement (reduction) of one point or more on the NLF severity scale. ${ }^{15}$ An additional analysis was conducted based on an improvement of two points or more from baseline (ie, achieving a score of 0 ("no wrinkle") or 1 ("shallow, just perceptible wrinkle"), again using the NLF severity scale.

For the subject's treatment preference at month 12, a one-sample test of proportions was employed with a null hypothesis of an equal (50\%) preference for missing data and the "no preference" response excluded from the analysis. 


\section{Results}

\section{Subjects}

A total of 80 subjects were enrolled, each receiving a single dose of the two study products into the right or left NLF, according to the randomization schedule. All subjects met the entry criterion of a score of 3 on the physician's NLF severity scale for each NLF. The mean age of the subjects was 47.5 years (range 33-69 years), with the majority of the study population being female (95\%) and Caucasian (95\%). A total of 77 (96\%) of the subjects completed the full 12-month study. Two subjects were lost to follow-up during the study (weeks 4 and 26, respectively), with a third subject withdrawing after 26 weeks due to a diagnosis of major depression, considered by the physician investigator to be unrelated to the treatment intervention.

\section{Dosing}

The physicians were permitted to administer up to two syringes each of the two study products into the allocated NLF, as stated previously. When analyzed, the mean volumes administered were similar: JUP $1.17 \mathrm{~mL}$ (range 0.6-1.6 mL) and PER $1.13 \mathrm{~mL}$ (range $0.6-2.0 \mathrm{~mL} ; P=0.22$ ). No supplementary administration ("touch-ups") was allowed after the treatment day (day 1).

\section{Efficacy}

\section{Primary outcome: one point or more improvement} on the NLF severity scale

At the first post-administration assessment (day 3), the physicians judged that both NLFs had improved by one grade or better on the NLF severity scale in all 80 subjects, consistent with a clinically relevant improvement with both fillers. The subjects' independent evaluation concurred, with 99\% (79/80) of NLFs treated with JUP and 95\% (76/80) of NLFs treated with PER $(P=0.08)$ judged by the subjects to have achieved this outcome (Table 1).

When evaluated again at the three subsequent clinic visits (day 7; months 1 and 3), there was again no difference between the two treatments in the proportion of NLFs that had maintained this one point or better correction, as evaluated by both the physicians and the subjects (Table 1 ; Figures 1 and 2).

However, at month 6, differences in the clinical performance began to consistently emerge, based on the ratings of both the physicians and subjects. At this clinic visit, this clinically relevant correction had been maintained in a significantly larger proportion of NLFs treated with

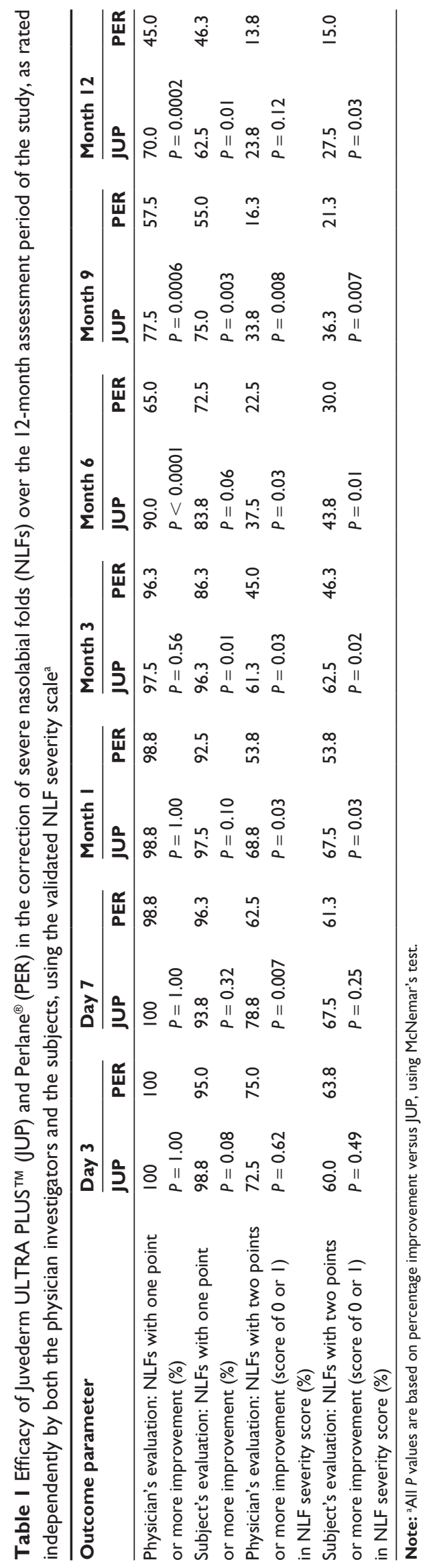




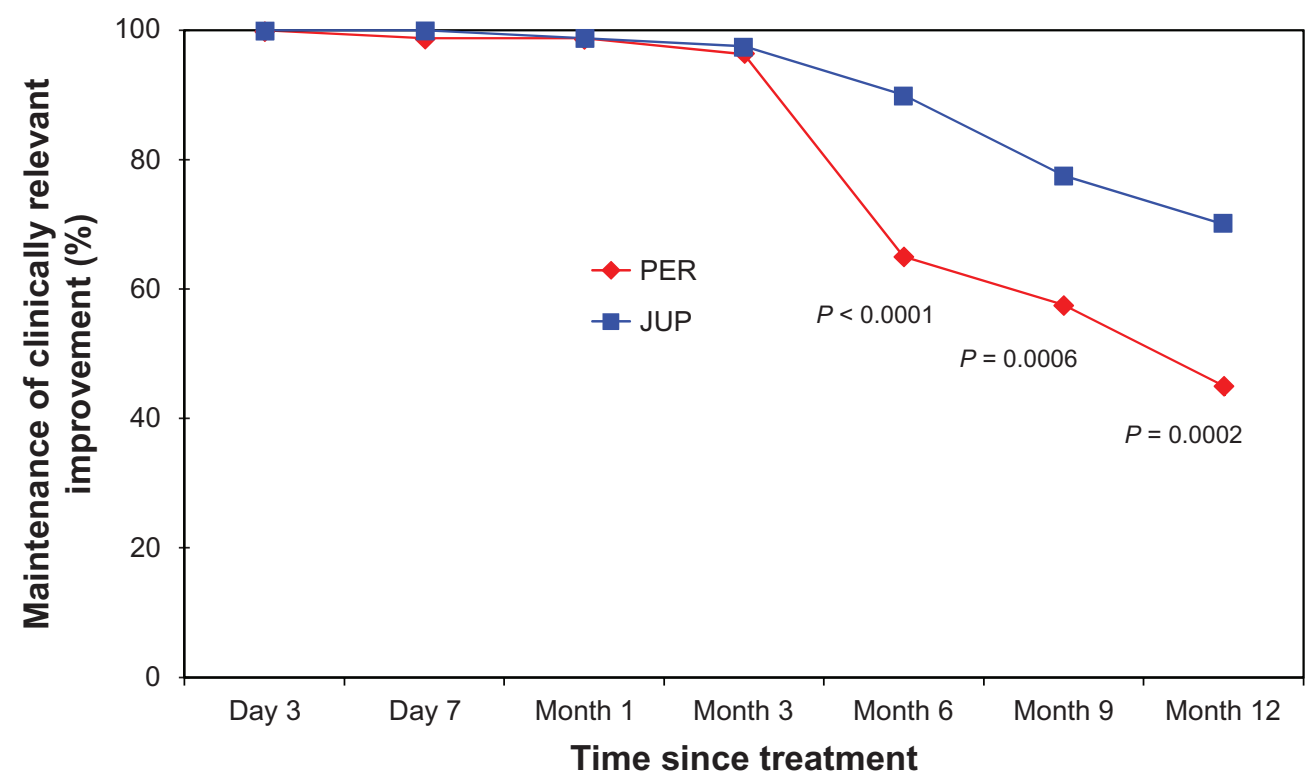

Figure I Proportion of nasolabial folds (NLFs) maintaining a clinically relevant improvement in the NLF severity score over the I2-month assessment period of the study (defined as: one point or greater improvement from the baseline score), as evaluated by the physician investigators. ${ }^{a}$

Note: aP values represent the difference between the two treatments from baseline, based on McNemar's test.

Abbreviations: JUP, Juvederm ULTRA PLUS ${ }^{\mathrm{TM}}$; PER, Perlane ${ }^{\circledR}$.

JUP, as documented by the physicians ( $90 \%$ vs $65 \%$; $P<0.0001)$, with a clear trend also evident based on the subjects' independent scoring ( $83.8 \%$ vs $72.5 \% ; P=0.06$ ). By the month 9 visit, a statistically significant difference between the two dermal fillers was documented by both the physicians and the subjects, demonstrating a greater relative longevity for JUP in the maintenance of the clinically relevant correction $(77.5 \%$ vs $57.5 \% ; P=0.0006$ and $75.0 \%$ vs $55.0 \% ; P=0.003$, respectively). This differential effect in favor of JUP was confirmed at the final clinical visit for the study (month 12), again as documented by both the physicians and the subjects $(70.0 \%$ vs $45.0 \%$; $P=0.0002$ and $62.5 \%$ vs $46.3 \% ; P=0.01$, respectively) (Table 1; Figures 1 and 2).

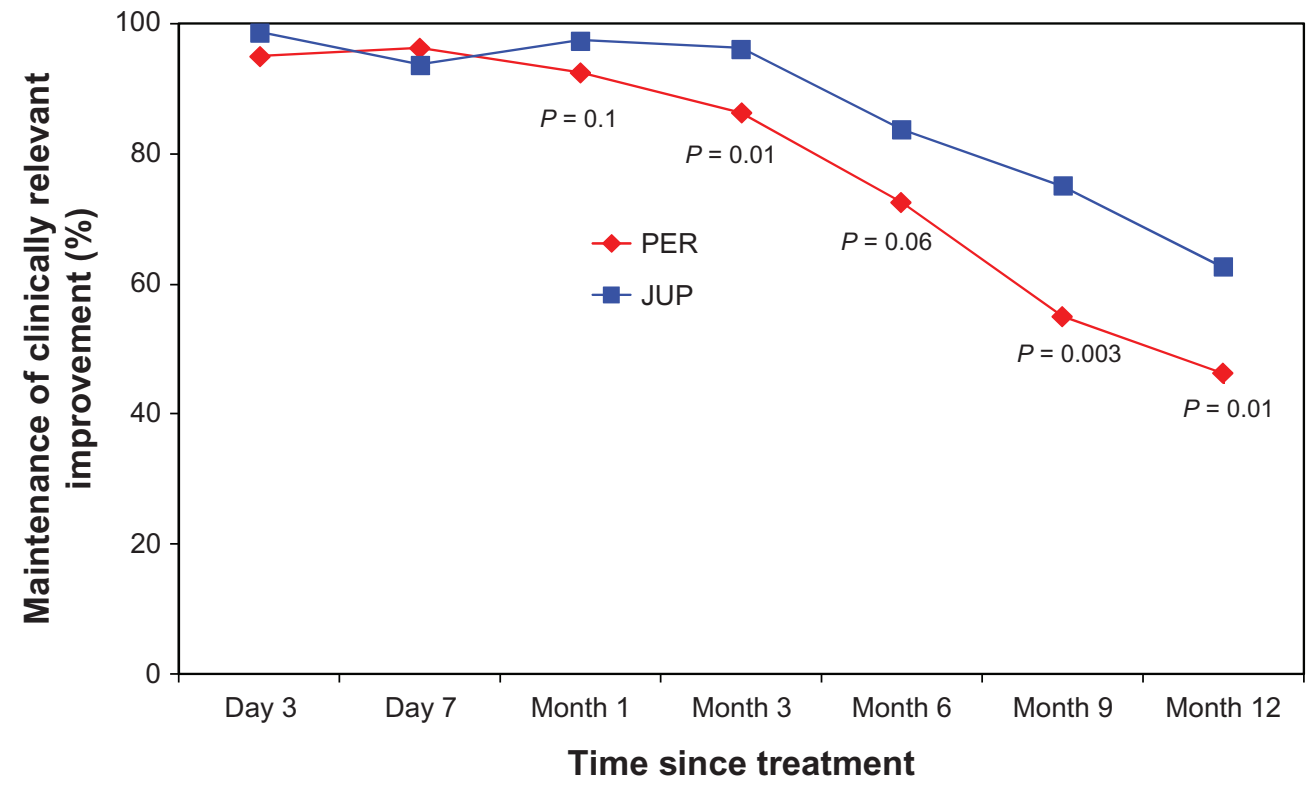

Figure 2 Proportion of nasolabial folds (NLFs) maintaining a clinically relevant improvement in the NLF severity score over the I2-month assessment period of the study (defined as: one point or greater improvement from the baseline score), as evaluated by the subjects. ${ }^{a}$

Note: aP values represent the difference between the two treatments from baseline, based on McNemar's test.

Abbreviations: JUP, Juvederm ULTRA PLUS ${ }^{\mathrm{TM}}$; PER, Perlane ${ }^{\circledR}$. 


\section{Secondary outcome: two points or greater improvement on NLF severity scale}

When the analysis was conducted for the more rigorous endpoint of two grades or more improvement (ie, improving to 0 ["no wrinkle"] or 1 ["shallow, just perceptible wrinkle"] on the NLF severity scale at day 3), the physicians and subjects also rated the treatments similarly at this clinic visit. However, by month 1, both the physicians and the subjects scored a significantly higher percentage of NLFs treated with JUP with a grade of 0 or 1 , with the investigators independently documenting this outcome with JUP from as early as day 7 . This differential effect for JUP was maintained for the remaining 11 months of the study (Table 1).

\section{Safety and tolerability}

Both treatments were well tolerated, with the majority of the treatment-related adverse events being transient, of mildto-moderate severity, and localized to the injection site. The individual parameters of pain, discomfort, or tenderness and other injection site reactions, as evaluated by the subject over the first 3 days postadministration, are summarized in this review and in Table 2. The physician's evaluation of bruising, erythema, and swelling on days 3 and 7 post-treatment are summarized in Table 3.

\section{Pain at injection site, postadministration (day I)}

As no anesthetic was used other than ice, the degree of pain following administration of the study treatments was rated by the subjects as moderate-to-severe. There was, however, no significant difference in the pain scores between the two study products as documented by the subjects on the elevenpoint VAS scale (Table 2).

\section{Assessment of discomfort and tenderness (days 3 and 7)}

The subjects reported a significantly higher degree of discomfort on palpation on day 3 following the administration of PER, as assessed on a 100-mm VAS. This difference was not apparent by day 7 . There was no difference reported between the two study treatments in the degree of tenderness on either day, again based on a 100-mm VAS (Table 2).

\section{Other injection site reactions}

Subject's evaluation (days I-3)

The subjects reported a significantly higher incidence of swelling and pain on days 1 and 2 on the side treated with PER, as documented in their diaries. There was, however, no statistical difference between the two treatments in the incidence of bruising or redness over these three post-treatment days. By day 3 , there was no difference between the two treatments for any of the injection site reactions (Table 2).

\section{Physician's evaluation (days 3 and 7)}

The physicians reported a significantly higher incidence of bruising and swelling on day 7 following JUP treatment. There was, however, no difference between the two treatments in the incidence of these events on day 3 or for the incidence of redness on either day according to the physicians (Table 3).

\section{Subjects' treatment preference}

At month 12, while still blinded to the study treatment allocation, the 77 of 80 subjects completing this clinic visit were asked to document the preferred study product by nominating the NLF site that they believed had the best treatment outcome. Over $71 \%$ of the subjects identified the side treated with JUP, whereas $21 \%$ nominated PER, and the remaining

Table 2 Incidence of injection site reactions (subject evaluation) ${ }^{\mathrm{a}}$

\begin{tabular}{|c|c|c|}
\hline Injection site reaction & JUP & PER \\
\hline $\begin{array}{l}\text { Postadministration pain } \\
\text { (Day I; eleven-point } \\
\text { Likert scale) (mean } \pm S D)\end{array}$ & $6.1 \pm 1.9$ & $5.8 \pm 2.1(P=0.23)$ \\
\hline \multicolumn{3}{|c|}{ Subject diaries (days I-3) } \\
\hline \multicolumn{3}{|l|}{ Presence of bruising (\%) } \\
\hline Day I & 70.1 & $71.4(P=0.80)$ \\
\hline Day 2 & 70.5 & $73.1(P=0.64)$ \\
\hline Day 3 & 63.3 & $59.5(P=0.56)$ \\
\hline \multicolumn{3}{|l|}{ Presence of redness (\%) } \\
\hline Day I & 62.8 & $62.8(P=1.00)$ \\
\hline Day 2 & 43.6 & $50.0(P=0.13)$ \\
\hline Day 3 & 26.6 & $30.4(P=0.44)$ \\
\hline \multicolumn{3}{|l|}{ Presence of swelling (\%) } \\
\hline Day I & 79.5 & $94.9(P=0.0005)$ \\
\hline Day 2 & 68.0 & $79.5(P=0.01)$ \\
\hline Day 3 & 51.9 & $55.7(P=0.44)$ \\
\hline \multicolumn{3}{|l|}{ Presence of pain (\%) } \\
\hline Day I & 61.3 & $76.9(P=0.009)$ \\
\hline Day 2 & 32.1 & $48.7(P=0.009)$ \\
\hline Day 3 & 16.5 & $24.1(P=0.11)$ \\
\hline \multicolumn{3}{|c|}{ Discomfort (I00 mm VAS) (mean \pm SD) } \\
\hline Day 3 & $7.3 \pm 12.8$ & $\mathrm{II} . \mathrm{I} \pm \mathrm{I} 6.5(P=0.03)$ \\
\hline Day 7 & $2.7 \pm 7.1$ & $2.8 \pm 9.6(P=0.83)$ \\
\hline \multicolumn{3}{|c|}{ Tenderness (I00 mm VAS) (mean \pm SD) } \\
\hline Day 3 & $9.9 \pm 12.6$ & $13.2 \pm 20.5(P=0.15)$ \\
\hline Day 7 & $3.8 \pm 8.8$ & $3.1 \pm 7.6(P=0.5)$ \\
\hline
\end{tabular}

Note: a $P$ values represent the difference between treatments based on McNemar's test.

Abbreviations: JUP, Juvederm ULTRA PLUS ${ }^{\mathrm{TM}}$; PER, Perlane ${ }^{\circledR}$; SD, standard deviation; VAS, visual analog scale. 
Table 3 Incidence of injection site reactions (physician evaluation)

\begin{tabular}{lll}
\hline Injection site reaction & JUP & PER \\
\hline $\begin{array}{l}\text { Presence of bruising (\%) } \\
\text { Day 3 }\end{array}$ & 61.5 & $60.0(P=0.67)$ \\
$\quad$ Day 7 & 28.8 & $11.3(P=0.00 \mathrm{I})$ \\
Presence of redness (\%) & & \\
$\quad$ Day 3 & 32.1 & $33.3(P=0.74)$ \\
$\quad$ Day 7 & 21.3 & $18.8(P=0.4 \mathrm{I})$ \\
Presence of swelling (\%) & & \\
$\quad$ Day 3 & 36.6 & $29.5(P=0.32)$ \\
Day 7 & 21.3 & $6.3(P=0.005)$ \\
\hline
\end{tabular}

Note: ${ }^{2} P$ values represent the difference between treatments based on McNemar's test. Abbreviations: JUP, Juvederm ULTRA PLUS ${ }^{\mathrm{TM}}$; PER, Perlane ${ }^{\circledR}$.

$8 \%$ of subjects reported no preference. When an analysis was conducted for subject preference for JUP versus PER only, this difference in favor of JUP was highly significant $(z=4.76, P<0.0001$; Figure 3$)$.

\section{Discussion}

When assessed at the first post-treatment assessment (day 3), both dermal fillers achieved an equivalent outcome, with a clinically relevant improvement documented in $100 \%$ of NLFs, as rated by the physician investigators and in $\geq 95 \%$ of NLFs, as determined independently by the subjects, based on the validated NLF severity scale. Further, a large proportion of the NLFs were rated by both the physicians ( $>72 \%$ ) and the subjects ( $\geq 60 \%$ ) with a score of 0 or 1 on day 3 , and this outcome was similar for both fillers (Table 1). This latter analysis was consistent with a complete or almost complete correction of the NLFs post-treatment, suggesting that optimal NLF correction was achieved in a large percentage of subjects following a single administration of these two fillers.

This initial equivalence in the correction of the NLFs, in addition to the similar mean volume administered and the consistent safety and tolerability profiles of these two fillers, enabled a fair comparison of their durability over the entire 12-month assessment period. Therefore, the greater relative longevity of the JUP in the maintenance of this clinically relevant correction, documented independently by both the physicians and the subjects after month 9 (based on one point or greater improvement), or after month 1 (for two points or greater improvement) on the NLF Severity Scale and preserved thereafter (Table 1; Figures 2 and 3), did not appear to be related to any specific differences in how the two fillers were initially administered.

A plausible explanation for this differential therapeutic effect observed in this study might be the distinct physiochemical properties of these two products, defined by their proprietary-based manufacturing technologies, affording JUP greater resistance to degradation by hyaluronidases and free radicals. The JUP gel is produced using a two-stage crosslinking process, resulting in a dense network of HA polymers that acts like a macromolecule. In comparison, PER consists of a relatively homogeneous gel mass of particles between $750 \mu \mathrm{m}$ and $1000 \mu \mathrm{m}$, produced by pushing the gel through a screen in the final production stage. Further, JUP has a higher

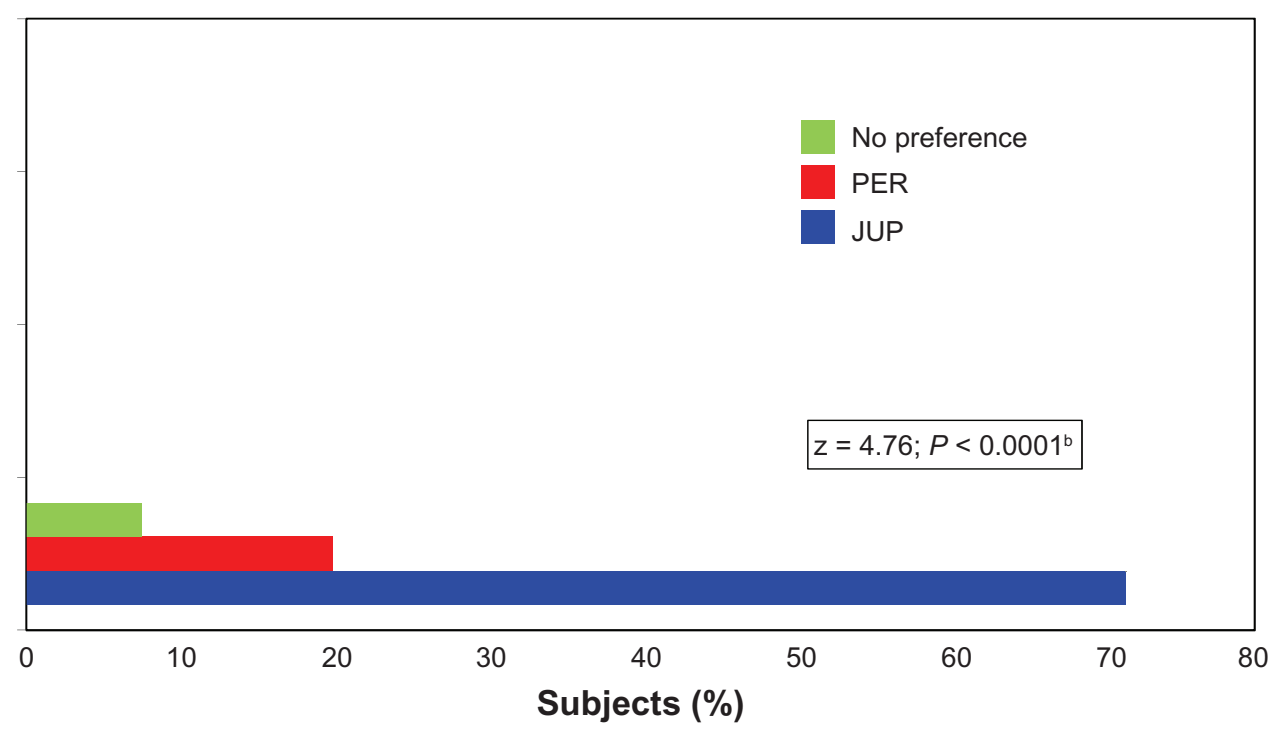

Figure 3 Treatment preference, as evaluated by the subjects ${ }^{a}$ at the end of the study (Month 12) while still blinded to the treatment allocation. Notes: a $77 / 80$ subjects who completed the I2-month study; ${ }^{\text {} P}$ value based on a difference in subject preference for Juvederm ULTRA PLUS ${ }^{T M}$ (JUP) versus Perlane ${ }^{\circledR}$ (PER) using a one-sample test of proportions with a null hypothesis of an equal $(50 \%)$ preference for missing data and a "no preference" response excluded from the analysis. Abbreviations: JUP, Juvederm ULTRA PLUS ${ }^{\mathrm{T}}$; PER, Perlane ${ }^{\circledR}$. 
relative degree of cross-linking to BDDE, which may create a more effective physical and chemical barrier to hyaluronase activity, thereby limiting access to its substrate, relative to the PER gel. Conversely, the distinct particles present in the PER gel may expose a greater relative surface area of its constituent HA to hydrolysis. ${ }^{10-13,16}$

The aforementioned hypothesis is consistent with the observations in a preceding in vitro study of the sensitivity of eleven HA gels to hyaluronase activity, in which the PER gel was hydrolyzed at a significantly faster rate than that for the JUP gel. ${ }^{14}$

In addition to the aforementioned, the higher relative HA concentration of JUP (24 mg/mL vs $20 \mathrm{mg} / \mathrm{mL}$ ) might convey a greater lifting ability through the binding of more water molecules, in comparison with the PER gel, which may also contribute to the relative longevity of JUP as observed in the current study. ${ }^{4,10,11}$

A potential weakness in the design of this study is the physician investigator's knowledge of the treatment allocation at the time of administration and thus the potential for bias during the outcome assessments over the ensuing 12 months. However, the randomization code at each site was kept in a separate location to the other study documentation and was therefore not freely accessible to the investigator after day 1. Further, as each investigator enrolled approximately 20 subjects, with all receiving both products, administered at similar mean volumes to the allocated NLFs, it would have been difficult for the investigator to recall the treatment randomization for any of the subjects over the 12-month period of the study. Notably, there was a consistency in the NLF scoring of the subjects and the physicians over the 12-month assessment (Table 1), with both groups independently recording their ratings on separate case report forms, which were not available to the other party. Finally, at the month 12 clinic visit, a majority (71.4\%) of the subjects nominated the NLF treated with JUP as their preferred treatment outcome while still blinded, further supporting the overall conclusions of this study.

\section{Conclusion}

In summary, the effectiveness of JUP, relative to PER, in the long-term maintenance of a clinically relevant correction of severe NLFs was clearly demonstrated in this 12-month within-subject study. As the mean volume administered into the allocated NLFs was virtually identical and the initial safety and efficacy of the two study products were very similar, a fair comparison of outcome for these two fillers is assumed. This greater apparent long-term durability of JUP may be related to its distinct physiochemical properties, affording it a greater relative resistance to enzymatic and/or free radical degradation in the dermis.

\section{Acknowledgments}

The study was sponsored by Allergan Australia. The project management, monitoring, and data management were performed independently by Omnicare Clinical Research Pty Ltd, Macquarie Park, NSW, Australia. The statistical analysis for this study was conducted independently by John Wlodarczyk Consulting Services, New Lambton, NSW, Australia.

\section{Disclosure}

GG, PB, MR, and RR were paid investigators and were provided with product by Allergan for use in this study. GG has also been a paid investigator for studies conducted on behalf of Kythera, Galderma, and Peplin. He has also been a paid consultant to Allergan, Galderma, Valeant, Peplin, and Q-Med. RR has been a paid investigator for studies conducted on behalf of Peplin and has been a paid consultant to Allergan, Galderma, and Peplin. JR and MH are paid employees of Allergan.

\section{References}

1. Ascher B, Coleman S, Alster T, et al. Full scope of effect of facial lipoatrophy: a framework of disease understanding. Dermatol Surg. 2006;32(8):1058-1069.

2. Sadick NS, Smoller B. A study examining the safety and efficacy of a fractional laser in the treatment of photodamage on the hands. J Cosmet Laser Ther. 2009;11(1):29-33.

3. Donofrio LM. Fat distribution: a morphologic study of the ageing face. Dermatol Surg. 2000;26:1107-1112.

4. Monheit GD, Thomas JA, Murphy DK, et al. Photographic documentation from a double-blind, randomized, multicenter study comparing new hyaluronic acid-based fillers vs crosslinked bovine collagen. Poster No. 202 presented at the Annual Scientific Meeting of the American Academy of Dermatology; July 26-30, 2006; San Diego, CA.

5. Coleman SR, Grover R. The anatomy of the aging face: volume loss and changes in 3-dimensional topography. Aesthet Surg J. 2006; 26(1S):S4-S9.

6. Mendelson BC, Jacobsone SR. Surgical anatomy of the midcheek: facial layers, spaces, and the midcheek segments. Clin Plast Surg. 2008;35(3):395-404; discussion 393.

7. Raspaldo H. Volumizing effect of a new hyaluronic acid sub-dermal facial filler: a retrospective analysis based on 102 cases. J Cosmet Laser Ther. 2008;10(3):134-142.

8. Kablik J, Monheit GD, Yu L, et al. Comparative physical properties of hyaluronic acid dermal fillers. Dermatol Surg. 2009;35 Suppl 1: 302-312.

9. Comper WD, Laurent TC. Physiological function of connective tissue polysaccharides. Physiol Rev. 1978;58(1):255-315.

10. Tezel A, Fredrickson GH. The science of hyaluronic acid dermal fillers. J Cosmet Laser Ther. 2008;10(1):35-42. 
11. Beasley KL, Weiss MA, Weiss RA. Hyaluronic acid fillers: a comprehensive review. Facial Plast Surg. 2009;25(2):86-94.

12. Monheit GD, Coleman KM. Hyaluronic acid fillers. Dermatol Ther. 2006;19(3):141-150.

13. Perlane US PI. FDA approved, February, 2009.

14. Sall I, Ferard G. Comparison of the sensitivity of 11 crosslinked hyaluronic acid gels to bovine testis hyaluronidase. Polym Degrad Stab. 2007;92:915-919.
15. Baumann LS, Shamban AT, Lupo MP, et al. Comparison of smooth-gel hyaluronic acid dermal fillers with cross-linked bovine collagen: a multicenter, double-masked, randomized, within-subject study. Dermatol Surg. 2007;33(Suppl 2):S128-S135.

16. Jones D, Tezel A, Borrell M. In vitro resistance to degradation of hyaluronic acid dermal fillers by ovine testicular hyaluronidase. Dermatol Surg. 2010;36:804-809.

\section{Publish your work in this journal}

Clinical, Cosmetic and Investigational Dermatology is an international, peer-reviewed, open access, online journal that focuses on the latest clinical and experimental research in all aspects of skin disease and cosmetic interventions. All areas of dermatology will be covered; contributions will be welcomed from all clinicians and basic science researchers globally. This journal is indexed on CAS. The manuscript management system is completely online and includes a very quick and fair peer-review system, which is all easy to use. Visit http://www.dovepress.com/testimonials.php to read real quotes from published authors.

Submit your manuscript here: http://www.dovepress.com/clinical-cosmetic-and-investigational-dermatology-journal 